\title{
The Role of De Novo T790M Mutation in The Origin of T790M Resistance Clone and in The Clinical Outcomes For Advanced EGFR-Mutant NSCLC Patients Receiving EGFR-TKIs
}

\section{Mei-Fang Li ( $\square$ 362952772@qq.com )}

Fujian Provincial Cancer Hospital https://orcid.org/0000-0002-5204-8386

Jing-Hui Lin

Fujian Provincial Cancer Hospital

Jing Zhang

Fujian Provincial Cancer Hospital

\section{Yun-Jian Huang}

Fujian Provincial Cancer Hospital

\section{Sheng-Chi Chen}

nanping first hospital

\section{Yan Wang}

nanping first hostipal

\section{Zhi-Yong He}

Fujian Provincial Cancer Hospital

\section{Research}

Keywords: de novo T790M, ddPCR, EGFR-TKI, resistance model, evolution

Posted Date: March 25th, 2021

DOI: https://doi.org/10.21203/rs.3.rs-338298/v1

License: (c) (1) This work is licensed under a Creative Commons Attribution 4.0 International License. Read Full License 


\section{Abstract}

Background: Increasing evidence suggests that de novo T790M mutation occurs at a low frequency in patients with epidermal growth factor receptor (EGFR)-mutant non-small cell lung cancer (NSCLC). However, the effects of this mutation on the formation of T790M resistant clones and efficacy of EGFR tyrosine kinase inhibitors (TKIs) remain unclear.

Methods: Fifty-nine treatment-naïve in-patients with advanced EGFR-mutant NSCLC were enrolled in this study between 2017 and 2018. We dynamically monitored T790M mutation in ctDNA of patients before and during treatment with first-generation EGFR-TKIs, which were administered every 2 to 3 months until disease progression.

Results: Among the patients, $28.81 \%$ (17/59) had a low-frequency de novo T790M mutation, $66.67 \%$ $(10 / 15)$ of them retained T790M mutation and resistance in this group was defined as "selection" resistance. T790M mutation was detected after treatment in $42.3 \%(11 / 26)$ of patients without de novo T790M mutation who experienced disease progression and resistance in this group was defined as "acquisition" resistance. After treatment with third-generation EGFR-TKI, patients with the "selection" T790M resistance mutation had significantly better objective response rate (ORR) and longer progressionfree survival (PFS) than those with the "acquisition" T790M resistance mutation.

Conclusion: Our study provides evidence that low-frequency de novo T790M mutation is not rare in patients with advanced EGFR-mutant NSCLC. T790M resistance mutations can have two origins: the selection of low-frequency de novo T790M clones or the acquisition of the mutation in initially T790Mnegative cells clinically. Since the origin of T790M resistance mutations can affect the efficacy of thirdgeneration EGFR-TKIs, these EGFR-TKIs may be more effective for the treatment of NSCLC patients with "selection" T790M resistance mutations.

\section{Background}

Over the past decade, tremendous progress has been made in the treatment of advanced non-small cell lung cancer (NSCLC), such as the use of tyrosine kinase inhibitors (TKIs) and molecularly targeted therapy for NSCLC patients with epidermal growth factor receptor (EGFR) mutations [1-5]. However, most patients that initially respond to EGFR-TKIs will eventually become drug resistant. The development of drug resistance to EGFR-TKIs has been attributed to EGFR T790M mutation ( $p$. Thr790Met) in approximately $50 \sim 60 \%$ of cases [5-9].

A two-model hypothesis has been proposed for the origin of EGFR T790M resistance clones from an evolutionary perspective, namely "selection" and "acquisition" models [10]. Under EGFR-TKI treatment pressure, a clone with a low mutant allele frequency (MAF) for de novo T790M can develop into a tumor with a dominant drug-resistant mutation. This is known as the "selection" resistance model. An EGFRactivating mutant clone that undergoes various processes such as the activation of the insulin-like growth factor 1 receptor (IGF-1R) signaling pathway, an altered chromatin state, or nuclear factor kappa B 
(NF-kB) activation $[11,12]$ and finally evolves into a T790M resistance clone is known as an "acquisition" resistance clone. Basic research and retrospective data have revealed differences in the effects of firstgeneration EGFR-TKIs and the sensitivity of T790M resistance clones to third-generation EGFR-TKIs between patients with "selection" and "acquisition" resistance mutations $[10,13,14]$.

At present, the probability of detecting de novo T790M mutation varies according to the detection samples and methods used. More than a decade ago, Michio et al [15] used direct sequencing and mutant-enriched polymerase chain reaction (PCR) to identify T790M mutations in small fractions of tumor cells collected from NSCLC patients before treatment. De novo T790M mutation, which may be one of the main origins of the "selection resistance clone," was found in only $0.3 \%-3 \%$ of NSCLC patients. However, the prevalence of de novo T790M mutation (ranges from $20 \%$ to $80 \%$ ) has been clearly underestimated due to the sensitivity of the detection methods available. These techniques include matrix-assisted laser desorption ionization-time of flight mass spectrometry (MALDI-TOF MS), TaqMan assay, colony hybridization, peptide nucleic acid (PNA)-clamping PCR, and scorpion amplified refractory mutation system (ARMS). Droplet digital PCR (ddPCR) is a novel method that can detect rare mutations with ultra-sensitive quantification [13,14, 16-24]. De novo T790M has low frequency and the highest reported MAF for the mutation is between $0.01 \%$ and $0.1 \%$ [14, 21-23]. However, the effect of lowfrequency de novo T790M mutation on the biological behavior of NSCLC patients with EGFR-activating mutations remains unclear. Therefore, detecting de novo T790M mutation and understanding the differences between the two resistance models are of great clinical significance for the first-line treatment of EGFR-activating mutant NSCLC and overcoming drug resistance.

To this end, we used ddPCR to monitor the EGFR T790M mutation in circulating tumor DNA (ctDNA) using serial plasma samples from advanced EGFR-mutant NSCLC patients before and during treatment with first-generation EGFR-TKIs. Using this approach, we evaluated the prevalence of de novo T790M mutation, the influence of the de novo T790M clone on "selection" and "acquisition" resistance models, and the differences in the effects of third-generation EGFR-TKIs on T790M resistance clones with different origins.

\section{Methods}

\section{Patients and samples}

A total of 59 treatment-naïve, advanced NSCLC patients with EGFR-activating mutation were enrolled in this study. The patients were admitted to the Fujian Provincial Cancer Hospital (Fuzhou, China) and The First Hospital of Nanping City, Fujian Province (China) during the period from March 10, 2017 to November 28, 2018. Analysis of EGFR-activating mutations in biopsy specimens from the patients was performed using ARMS-PCR or next generation sequencing (NGS). Furthermore, EGFR T790M mutation in the ctDNA of the patient plasma samples was assessed every 2 to 3 months using ddPCR before and during first-generation EGFR-TKI treatment. However, serial plasma ctDNA samples were only collected from a limited number of patients. Based on the results, the eligible patients were stratified into two 
groups: EGFR-activating mutation with and without the low-frequency de novo T790M mutation at the baseline.

\section{Treatment regimens and response evaluation}

All participants received $250 \mathrm{mg}$ Gefitinib (AstraZeneca Pharmaceuticals; Waltham, MA, USA) once a day (QD) or 150 mg Icotinib (Betta Pharmaceuticals Co., Ltd.; Hangzhou, China) three times a day (TID). Some of the patients also received $80 \mathrm{mg}$ of the third-generation EGFR-TKI Osimertinib (AstraZeneca Pharmaceuticals; Waltham, MA, USA) QD when EGFR T790M mutation was detected in their plasma ctDNA or biopsy specimens after disease progression. The Response Evaluation Criteria in Solid Tumor (RECIST version 1.1) was used to evaluate the treatment responses of the patients. Progression-free survival 1 (PFS1) was recorded after treatment with first-generation EGFR-TKIs in patients with T790M resistance mutation if the disease progressed. PFS2 represented the length of survival for all patients after treatment with first-generation EGFR-TKIs. PFS3 represented the length of survival after treatment with the third-generation EGFR-TKI until disease progression.

\section{Droplet Digital PCR (ddPCR)}

ddPCR was performed following the manufacturer's protocols; therefore, a minimum of $8 \mathrm{ml}$ of peripheral blood was required. The presence of the EGFR T790M mutation in the samples was determined using the QX200 ddPCR System (Bio-Rad, Marnes-la-Coquette, France). Qiamp Circulating Nucleic Acid Kits were obtained from Qiagen (Cat NO. 55114) and used for the purification of free-circulating DNA in the plasma or serum. The quality of the extracted nucleic acid was determined using a Qubit Fluorometer. The samples were first emulsified using a QX200 droplet generator, then transferred to 96-well plates and amplified in a thermal cycler with the following thermal profile: $42^{\circ} \mathrm{C}$ for $5 \mathrm{~min} ; 94^{\circ} \mathrm{C}$ for $5 \mathrm{~min} ; 8$ cycles of $94^{\circ} \mathrm{C}$ for $15 \mathrm{~s}, 60^{\circ} \mathrm{C}$ for $25 \mathrm{~s}$, and $72^{\circ} \mathrm{C}$ for $40 \mathrm{~s}$; and $72^{\circ} \mathrm{C}$ for $5 \mathrm{~min}$. The fluorescence signals from individual sample droplets in each well were measured with a QX200 droplet reader. The MAF relative to the frequency of the wild-type allele was analyzed using QuantaSoft V.1.7.4.0917 software. The MAF was determined according to Poisson's distribution principle and the number and proportion of positive droplets. Positive and negative cut-off points were established for the controls. The cut-off point for positive droplets was set at three droplets based on preliminary assays and the manufacturer's protocols. This kit is very sensitive and can detect $0.33 \%$ of EGFR gene mutant DNA in patient samples.

\section{Statistical analysis}

The clinicopathologic variables were compared using Fisher's exact test or the Chi-squared test. PFS was analyzed using the Kaplan-Meier method and the differences in PFS between groups were compared using the log-rank test. In the tests, a two-sided P value (less than 0.05 ) was considered statistically significant. The statistical software SPSS version 23.0 (SPSS, Inc.; Chicago, IL, USA) was used for all statistical analyses in this study.

\section{Informed consent and ethical approval}


The protocol for this study was reviewed and approved by the Ethical Review Committee of Fujian Provincial Cancer Hospital (approval no.2017-069-01). Written informed consent with a detailed description of the study was obtained from all participants.

\section{Results}

\section{Clinicopathological characteristics of patients with EGFR mutation}

Fifty-nine advanced EGFR-mutant NSCLC patients were enrolled in this study, comprising 28 men and 31 women with a median age of 56 years (range, 38-79 years) at diagnosis. Among these patients, $98.3 \%$ were diagnosed with adenocarcinoma and $94.9 \%$ had an Eastern Cooperative Oncology Performance Status (ECOG-PS) score of 0 or 1 . Nineteen of the patients had brain metastases and $32.2 \%$ were at stage $\triangle A$ at baseline. Thirty-four patients harbored an EGFR exon 19 deletion mutation and the remaining 25 patients harbored an EGFR exon 21 L858R mutation. Using ddPCR, T790M mutation in the ctDNA samples from all 59 patients was assessed before treatment. Based on the ddPCR results, the patients were stratified into two groups: 17 patients had an EGFR-activating mutation with low-frequency de novo T790M mutation and the other 42 patients were T790M-negative pretreatment. Most of the baseline clinicopathological features such as gender, age, ECOG-PS score, smoking status, stage, and brain metastases were similar between the two groups. However, the composition of the EGFR-activating mutation differed between the groups. As shown in Table 1, the low-frequency de novo T790M mutation may coexist with EGFR 19del mutation $(\mathrm{p}=0.020)$.

\section{Low-frequency de novo T790M mutation is not rare in NSCLC patients with EGFR-activating mutation}

T790M mutation in the ctDNA samples from all 59 patients was evaluated with ddPCR before treatment. The results showed that the overall incidence of low-frequency de novo T790M mutation was $28.81 \%$ (17/59). The MAF for T790M mutation ranged from $0.01 \%$ to $0.28 \%$, with an ultra-low allele frequency between $0.01 \%$ and $0.11 \%$ in approximately $88.24 \%$ (15/17) of patients (Figure 1$)$.

\section{MAF of plasma T790M mutation showed an upward trend during treatment with first-generation EGFR- TKls}

Serial plasma samples were collected every 2 or 3 months during treatment with first-generation EGFRTKIs, although plasma samples could not be collected at every visit for all patients. A total of 214 plasma samples were collected for T790M detection using ddPCR, among which 59 plasma samples were obtained pretreatment and 155 plasma samples were obtained during the first-generation EGFR-TKI treatment. A downward trend in the MAF of T790M was found in most patients with a low-frequency de novo T790M mutation at the early stage of EGFR-TKI treatment, but an upward trend was observed when the tumor progressed. After disease progression, nine patients retained T790M mutation in their ctDNA, which was detected by ddPCR. As shown in Figure 2, the MAF of T790M resistance mutation ranged from $0.04 \%$ to $1.75 \%$. Furthermore, in the group without de novo T790M mutation pretreatment, the MAF of T790M was negative at the early stage of EGFR-TKI treatment. However, the MAF of T790M in most patients without de novo T790M mutation pretreatment also showed an upward trend over the course of 
tumor development. ddPCR results showed T790M mutation in nine patients after disease progression and the MAF for T790M resistance mutation ranged from $0.034 \%$ to $12.9 \%$ (Figure 3 ).

\section{Most cases of T790M resistance mutation emerge due to the selection of the low-frequency de novo T790M clone}

In this study, EGFR T790M mutation in the plasma ctDNA of the patients was monitored with ddPCR every 2 to 3 months until disease progression during treatment with first-generation EGFR-TKIs. In the group with the low-frequency de novo T790M mutation, 15 patients exhibited disease progression and $66.67 \%$ (10/15) of them retained the T790M mutation, which was detected with NGS in biopsy specimens from one patient and with ddPCR in ctDNA samples from nine patients. The resistance model for the above-mentioned cases was defined as "selection" resistance. In the group without T790M pretreatment, 26 patients exhibited disease progression and $42.3 \%(11 / 26)$ of them were found to have developed T790M resistance mutation. T790M mutation was detected in ctDNA samples from nine of these patients using ddPCR and in biopsy specimens from the other two patients using ARMS-PCR. The resistance model for these patients was defined as "acquisition" resistance. Taken together, the probability of T790M mutation was $51.22 \%(21 / 41)$ in all patients after disease progression (Figure 4).

\section{There may be no difference in the occurrence time of T790M resistance mutation with "selection" and "acquisition" origins in clinical practice}

As shown in Figure 5, among the patients with the T790M resistance mutation after disease progression during the first-generation EGFR-TKI treatment, those with low-frequency de novo T790M mutation had a median PFS1 of 11.0 months (95\% confidence interval [CI], 9.141-12.859 months). Patients without the T790M mutation before treatment had a median PFS1 of 11.5 months $(95 \% \mathrm{Cl}, 9.989-13.011$ months; $p=$ 0.649). The median PSF1 for both groups was similar, suggesting there may be no difference in the occurrence time of T790M resistance mutation between patients with "selection" and "acquisition" resistance mutations in clinical practice.

\section{Low-frequency de novo T790M mutation may not affect the efficacy of first-generation EGFR-TKls}

The median follow-up period for the patients in this study was 21.3 months (range, 1.2-36.2 months). At the end of the follow-up period (Mar 23, 2020), 88.2\% (15/17) of patients with a low-frequency de novo T790M mutation experienced disease progression, with an objective response rate (ORR) of $82.4 \%$. In the group of patients (42 cases) without pretreatment de novo T790M mutation, six patients were lost to follow-up and $72.2 \%$ (26/36 cases) of the remaining patients developed disease progression, with an ORR of 83.3\%. As shown in Figure 6, patients with low-frequency de novo T790M mutation had a median PFS2 of 12.0 months (95\% Cl, 9.781-14.219 months), which was comparable to that of patients without pretreatment T790M (median PFS2, 11.4 months; $95 \% \mathrm{Cl}$, 9.342-13.458 months; $p=0.451$ ).

The "selection" model of T790M resistance mutation may be treated more effectively with thirdgeneration EGFR-TKls 
In the group of patients with the low-frequency de novo T790M mutation, 10 patients retained the T790M mutation after disease progression. Seven of these patients were further treated with Osimertinib. The optimal efficacy in this group was estimated and indicated a partial response (PR) in six patients (85.7\%) and stable disease (SD) in one patient (14.3\%), with an ORR of $85.7 \%$. In this group, four patients experienced disease progression at the end of the follow-up period and the PFS3 for five patients was more than 8 months. In the group of patients without T790M mutation before treatment, nine patients further received Osimertinib when T790M mutation was discovered after disease progression. The optimal efficacy in this group was estimated as PR in two (25.0\%) patients and SD in six $(75.0 \%)$ patients, with an ORR of $25.0 \%$. Six patients experienced disease progression at the end of the follow-up period and the PFS3 for five patients was less than 6 months in this group (Figure 7,8).

\section{Discussion}

EGFR T790M mutation has been widely studied and is commonly recognized as a drug resistance mutation after treatment with first or second-generation EGFR-TKIs [25-28]. However, the understanding of de novo T790M mutation in patients with advanced NSCLC before EGFR-TKI treatment is insufficient. Increasing evidence suggests that de novo T790M mutation exists in NSCLC patients with EGFRactivating mutations. Due to the differences in detection methods and samples, the occurrence probability of de novo T790M mutation was variable in previous reports [13, 14, 16-22]. ddPCR is the most sensitive molecular detection method for de novo T790M mutation at present and its analytical sensitivity is close to $0.001 \%[14,29]$. Masaru et al [21] used ddPCR to assess T790M mutation and found de novo T790M mutation in 79.9\% (298/373 cases) of surgically resected tumor specimens from patients with EGFR-activating mutations. The MAF of de novo T790M mutation in these patients ranged from $0.009 \%$ to $26.9 \%$, with an ultra-low allele frequency between $0.01 \%$ and $0.1 \%$ in approximately $80 \%$ of cases. However, there are few reports on the detection rate for de novo T790M mutation in plasma samples from patients using ddPCR. Hence, we evaluated the occurrence of de novo T790M mutation in the plasma ctDNA of advanced NSCLC patients with EGFR-activating mutations using ddPCR. The results showed that the overall incidence of de novo T790M mutation was $28.81 \%$, with a MAF ranging from $0.01 \%$ to $0.28 \%$ and an ultra-low allele frequency between $0.01 \%$ and $0.11 \%$ in approximately $88.24 \%$ of cases. The difference in the prevalence of de novo T790M mutation between our results and other studies can be attributed to differences in the samples and the limited size of the tested populations. However, the MAF of de novo T790M mutation in our study was similar to those in previous reports.

The most common EGFR-activating mutations are EGFR 19del and 21L858R [5], but the probability of pretreatment de novo T790M that coexists with 19del or 21L858R remains controversial. Several reports showed that the de novo T790M mutation likely coexists with EGFR 21L858R mutation [24, 30-34]. However, in our study, we found that de novo T790M was more likely to coexist with 19del mutation, which was consistent with the report by Julie A et al [14]. Potential explanations for the differences between our results and other reports include our small sample size and inconsistent detection methods for T790M mutation. Therefore, further studies are needed to understand the differences in the de novo T790M mutation between EGFR 19del and 21L858R mutations in NSCLC. 
Although the existence of de novo T790M has been recognized, the effect of the mutation on the efficacy of first-generation EGFR-TKIs was inconsistent in previous reports. In our study, patients with and without de novo T790M mutation had a PFS of 12.0 vs 11.4 months after treatment with first-generation EGFRTKIs, respectively $(p=0.451)$. Linda Ye et al [23] reported similar results. In previous reports $[13,16,20$, 35], EGFR-activating mutant NSCLC patients harboring the de novo T790M mutation who received firstgeneration EGFR-TKI treatment had a shorter PFS than those without de novo T790M. Two meta-analysis studies also support these observations [36,37]. On the contrary, several reports suggested that patients with de novo T790M mutation had a longer median PFS than T790M-negative patients [38-40]. Yoshihiko et al [18] evaluated patients with strongly positive T790M mutation signals and those with modestly positive signals and found that the former had a longer PFS $(p=0.0019)$, suggesting that the MAF of de novo T790M mutation may impact the effect of EGFR-TKIs. Julie A et al [14] detected de novo T790M using ddPCR and defined a specific threshold of $0.1 \%$ for the MAF, which separated patients with longer PFS from those with EGFR-activating mutations $(p=0.029)$. Therefore, it is necessary to establish a threshold for low-frequency de novo T790M mutation in future clinical practice in order to determine the prognostic value of de novo T790M for NSCLC patients with EGFR-activating mutation.

There is a two-model hypothesis (i.e. the "selection" and "acquisition" resistance models) for the origin of T790M resistance clones during disease progression after first-generation EGFR-TKIs treatment. However, there is little direct clinical evidence to support the existence of tumor sub-clones before treatment as the main mechanism for the eventual evolution of drug-resistant clones. In our study, 66.67\% (10/15) of patients in the group with low-frequency de novo T790M retained the T790M mutation after disease progression, while $42.31 \%$ (11/26) of patients without the T790M mutation before treatment developed the T790M resistance mutation after disease progression. In other words, most of the T790M resistance mutations developed due to the evolution of a T790M sub-clone that existed pretreatment and the lowfrequency de novo T790M mutation may be primarily responsible for the subsequent development of the T790M resistance clone.

As described above, the T790M resistance clone can develop from two pathways, namely the selection of a pre-existing EGFR T790M-positive clone or via the evolution of drug-tolerant cells (DTCs) that are initially EGFR T790M-negative [10,41]. Previous studies showed that the time of development for druginduced T790M resistance clones was different in the two models. The "acquisition" of T790M resistance clone requires a longer drug-induced period because the tumor progresses through a drug-tolerant phase and later evolves into a drug-resistant state [10,12,41,42]. However, the clinical evidence is insufficient to show the difference in the occurrence time of T790M resistance mutation. In all of the patients with the T790M resistance mutation when the disease progressed, we found no differences in the PFS after treatment with first-generation EGFR-TKIs between the patients with and without the de novo T790M mutation (median PFS, 11.0 vs. 11.5 months, respectively; $p=0.649$ ). Therefore, our clinical data did not confirm the existence of a difference in the emergence of the T790M drug-resistant clone in the two models and further investigations with laboratory models and clinical data are required. 
The "acquisition" model of T790M resistance evolves from T790M-negative DTCs after the drug-tolerant stage $[10,12,41,42]$. This evolutionary process makes the tumor cells more heterogeneous, resulting in less sensitivity in the "acquisition" T790M resistance clone to the follow-up drugs. In-vitro studies showed that the acquisition of T790M resistance from DTCs leads to a low level of apoptosis, which can be increased by the over-expression of Bcl-2-like protein 11 (BIM) [10, 43, 44]. Our study showed similar results, as patients with the "selection" T790M resistance mutation had better ORR to Osimertinib than those with the "acquisition" T790M resistance mutation (ORR, $85.7 \%$ vs $25.0 \%$ ). Although we found no statistically significant difference between the PFS in the two groups due to the small sample size in our study, the PFS of most patients with the "acquisition" T790M resistance mutation was less than 6 months after Osimertinib treatment. This suggests that the origin of the T790M resistance mutation may affect the efficacy of third-generation EGFR-TKIs and that the low-frequency de novo T790M mutation is a good prognostic marker for treatment with third-generation EGFR-TKIs among patients with T790M resistance mutation.

Although EGFR-TKIs benefit the survival of patients with EGFR-activating mutations, more individual and detailed management of patients is still required. It remains unclear whether dynamic detection of ctDNA T790M is necessary before and during treatment with first-generation EGFR-TKIs. In our study, most of the MAF for T790M mutation in patients with and without de novo T790M mutation showed an upward trend during tumor progression but had no regularity. D. Zheng et al [45] reported that T790M ctDNApositivity was confirmed in some patients at a median of 2.2 months prior to clinical disease progression following treatment with first-generation EGFR-TKIs. However, there is no clinical evidence to support the survival benefits of intervention with third-generation EGFR-TKIs in advance for patients with ctDNA T790M positivity or those with an increasing MAF. The AZD9291 (Osimertinib) Treatment on Positive PLasma T790M in EGFR-mutant NSCLC Patients (APPLE) trial assessed the dynamic predictive value of liquid biopsies to confirm the appropriate timing for Osimertinib administration to patients with either positive ctDNA T790M or disease progression according to RECIST [46]. However, the results of this trial have not been announced. The detection of T790M in serial plasma samples may be used to predict the progression of tumors and reverse resistance to first-generation EGFR-TKIs ahead of time. Osimertinib has been recommended as one of the first-line treatment options for NSCLC patients with EGFRactivating mutations. However, due to the potency ratio and other factors, only some patients are eligible for third-generation EGFR-TKIs as the first-line treatment. In NSCLC patients with both EGFR-activating mutation and de novo T790M, better curative effects can be achieved by eliminating the secondary mutation and reducing tumor heterogeneity at the beginning of tumor treatment if the third generation of EGFR-TKIs can be used as the first-line treatment. For example, the AZENT trial (ClinicalTrialsals.gov, NCT02841579) was performed to evaluate the efficacy and safety of Osimertinib as a first-line treatment for advanced NSCLC patients with de novo T790M mutation, indicating the importance of de novo T790M in tumor therapy.

\section{Conclusion}


Low-frequency de novo T790M mutation is common in patients with EGFR-activating mutant NSCLC. The use of ultra-sensitive detection methods such as ddPCR to evaluate de novo T790M mutation is of clinical value for the individualized management of advanced NSCLC patients with EGFR-activating mutation because of the role of this mutation in not only predicting the prognosis of patients but also in the choice of treatment strategies. As previously reported in the literature, de novo T790M mutation may have an effect on the treatment efficacy of first-generation EGFR-TKIs. In order to achieve better efficacy, third-generation EGFR-TKIs may be selected as the first-line treatment for advanced NSCLC patients with both EGFR-activating mutations and de novo T790M mutation. Furthermore, the origin of the T790M resistance mutation might affect the efficacy of third-generation EGFR-TKIs in patients with "acquisition" T790M resistance mutation because of a reduced apoptotic response in the tumor cells to thirdgeneration EGFR-TKIs. Therefore, the combination of third-generation EGFR-TKIs with anti-apoptosis inhibitors may be a good approach in clinical practice.

\section{Abbreviations}

epidermal growth factor receptor (EGFR), non-small cell lung cancer (NSCLC), tyrosine kinase inhibitors (TKIs), objective response rate (ORR), progression-free survival (PFS), mutant allele frequency (MAF), insulin-like growth factor 1 receptor (IGF-1R), nuclear factor kappa B (NF-kB), polymerase chain reaction (PCR), matrix-assisted laser desorption ionization-time of flight mass spectrometry (MALDI-TOF MS), peptide nucleic acid (PNA), amplified refractory mutation system (ARMS), droplet digital PCR (ddPCR), circulating tumor DNA (ctDNA), next generation sequencing (NGS), once a day (QD), three times a day (TID), Eastern Cooperative Oncology Performance Status (ECOG-PS), partial response (PR), stable disease (SD), drug-tolerant cells (DTCs).

\section{Declarations}

\section{Ethics approval and consent to participate}

The protocol for this study was reviewed and approved by the Ethical Review Committee of Fujian Provincial Cancer Hospital (approval no.2017-069-01).

\section{Consent for publication}

With the consent for publication for all authors.

\section{Availability of data and material}

Not applicable

\section{Competing interests}

The authors declare that they have no competing interests. 


\section{Funding}

This study was supported by grants from the Startup Fund for Scientific Research, Fujian Medical University (grant no. 2017XQ1217).

\section{Authors' contributions}

Li Meifang carried out data curation, formal analysis, investigation, methodology, software, visualization and writing-original draft. Lin Jinghui participated in data curation, methodology and resources. Zhang Jing participated in methodology and resources. Huang Yunjian participated in methodology and resources. Chen Shengchi participated in methodology, resources and supervision. Wang Yan participated in methodology and resources. He Zhiyong carried out cconceptualization, data curation, funding acquisition, project administration, supervision and writing-review \& editing.

\section{Acknowledgements}

Not applicable

\section{Authors' information}

1Department of Thoracic Medical Oncology, Fujian Medical University Cancer Hospital, Fujian Cancer Hospital, Fuzhou 350014, Fujian Province, People's Republic of China 2 Fujian Provincial Key Laboratory of Translation Cancer Medicine, Fujian Medical University Cancer Hospital, Fujian Cancer Hospital, Fuzhou 350014, Fujian Province, People's Republic of China 3Department of Medical Oncology, Nanping First Hospital Affiliated to Fujian Medical University, Nanping 354200, Fujian Province, People's Republic of China

\section{References}

1. Mok TS, Wu YL, Thongprasert S, Yang CH, Chu DT, Saijo N, Sunpaweravong P, Han B, Margono B, Ichinose $Y$ et al: Gefitinib or carboplatin-paclitaxel in pulmonary adenocarcinoma. $N$ Engl J Med 2009, 361(10):947-957.

2. Maemondo M, Inoue A, Kobayashi K, Sugawara S, Oizumi S, Isobe H, Gemma A, Harada M, Yoshizawa $\mathrm{H}$, Kinoshita I et al: Gefitinib or chemotherapy for non-small-cell lung cancer with mutated EGFR. N Engl J Med 2010, 362(25):2380-2388.

3. Mitsudomi T, Morita S, Yatabe Y, Negoro S, Okamoto I, Tsurutani J, Seto T, Satouchi M, Tada H, Hirashima $T$ et al: Gefitinib versus cisplatin plus docetaxel in patients with non-small-cell lung cancer harbouring mutations of the epidermal growth factor receptor (WJTOG3405): an open label, randomised phase 3 trial. Lancet Oncol 2010, 11(2):121-128.

4. Langer CJ: Epidermal growth factor receptor inhibition in mutation-positive non-small-cell lung cancer: is afatinib better or simply newer? J Clin Oncol 2013, 31(27):3303-3306. 
5. Riely GJ, Yu HA: EGFR: The Paradigm of an Oncogene-Driven Lung Cancer. Clin Cancer Res 2015, 21(10):2221-2226.

6. Gainor JF, Shaw AT: Emerging paradigms in the development of resistance to tyrosine kinase inhibitors in lung cancer. J Clin Onco/2013, 31(31):3987-3996.

7. Yu PP, Vose JM, Hayes DF: Genetic Cancer Susceptibility Testing: Increased Technology, Increased Complexity. J Clin Oncol 2015, 33(31):3533-3534.

8. Yu HA, Arcila ME, Rekhtman N, Sima CS, Zakowski MF, Pao W, Kris MG, Miller VA, Ladanyi M, Riely GJ: Analysis of tumor specimens at the time of acquired resistance to EGFR-TKI therapy in 155 patients with EGFR-mutant lung cancers. Clin Cancer Res 2013, 19(8):2240-2247.

9. Finlay MR, Anderton M, Ashton S, Ballard P, Bethel PA, Box MR, Bradbury RH, Brown SJ, Butterworth S, Campbell A et al: Discovery of a potent and selective EGFR inhibitor (AZD9291) of both sensitizing and T790M resistance mutations that spares the wild type form of the receptor. J Med Chem 2014, 57(20):8249-8267.

10. Hata AN, Niederst MJ, Archibald HL, Gomez-Caraballo M, Siddiqui FM, Mulvey HE, Maruvka YE, Ji F, Bhang $\mathrm{H}-\mathrm{eC}$, Krishnamurthy Radhakrishna $\mathrm{V}$ et al: Tumor cells can follow distinct evolutionary paths to become resistant to epidermal growth factor receptor inhibition. Nature Medicine 2016, 22(3):262269.

11. Suda K, Bunn PA, Jr., Rivard CJ, Mitsudomi T, Hirsch FR: Primary Double-Strike Therapy for Cancers to Overcome EGFR Kinase Inhibitor Resistance: Proposal from the Bench. J Thorac Oncol 2017, 12(1):27-35.

12. Sharma SV, Lee DY, Li B, Quinlan MP, Takahashi F, Maheswaran S, McDermott U, Azizian N, Zou L, Fischbach MA et al: A chromatin-mediated reversible drug-tolerant state in cancer cell subpopulations. Cell 2010, 141(1):69-80.

13. Su K-Y, Chen H-Y, Li K-C, Kuo M-L, Yang JC-H, Chan W-K, Ho B-C, Chang G-C, Shih J-Y, Yu S-L et al: Pretreatment Epidermal Growth Factor Receptor (EGFR) T790M Mutation Predicts Shorter EGFR Tyrosine Kinase Inhibitor Response Duration in Patients With Non-Small-Cell Lung Cancer. Journal of Clinical Oncology 2012, 30(4):433-440.

14. Vendrell JA, Mazieres J, Senal R, Rouquette I, Quantin X, Pujol J-L, Roch B, Bouidioua A, Godreuil S, Coyaud E et al: Ultra-sensitive EGFRT790M Detection as an Independent Prognostic Marker for Lung Cancer Patients Harboring EGFRdel19 Mutations and Treated with First-generation TKIs. Clinical Cancer Research 2019, 25(14):4280-4289.

15. Inukai M, Toyooka S, Ito S, Asano H, Ichihara S, Soh J, Suehisa H, Ouchida M, Aoe K, Aoe M et al: Presence of Epidermal Growth Factor ReceptorGene T790M Mutation as a Minor Clone in NonSmall Cell Lung Cancer. Cancer Research 2006, 66(16):7854-7858.

16. Rosell R, Molina MA, Costa C, Simonetti S, Gimenez-Capitan A, Bertran-Alamillo J, Mayo C, Moran T, Mendez P, Cardenal F et al: Pretreatment EGFR T790M Mutation and BRCA1 mRNA Expression in Erlotinib-Treated Advanced Non-Small-Cell Lung Cancer Patients with EGFR Mutations. Clinical Cancer Research 2011, 17(5):1160-1168. 
17. Lee Y, Lee GK, Lee Y-S, Zhang W, Hwang J-A, Nam B-H, Kim SH, Kim J-H, Yun T, Han J-Y et al: Clinical outcome according to the level of preexisting epidermal growth factor receptor T790M mutation in patients with lung cancer harboring sensitive epidermal growth factor receptor mutations. Cancer 2014, 120(14):2090-2098.

18. Fujita Y, Suda K, Kimura H, Matsumoto K, Arao T, Nagai T, Saijo N, Yatabe Y, Mitsudomi T, Nishio K: Highly sensitive detection of EGFR T790M mutation using colony hybridization predicts favorable prognosis of patients with lung cancer harboring activating EGFR mutation. J Thorac Oncol 2012, 7(11):1640-1644.

19. Costa C, Molina MA, Drozdowskyj A, Gimenez-Capitan A, Bertran-Alamillo J, Karachaliou N, Gervais R, Massuti B, Wei J, Moran T et al: The impact of EGFR T790M mutations and BIM mRNA expression on outcome in patients with EGFR-mutant NSCLC treated with erlotinib or chemotherapy in the randomized phase III EURTAC trial. Clin Cancer Res 2014, 20(7):2001-2010.

20. Maheswaran S, Sequist LV, Nagrath S, Ulkus L, Brannigan B, Collura CV, Inserra E, Diederichs S, lafrate AJ, Bell DW et al: Detection of mutations in EGFR in circulating lung-cancer cells. $N$ Engl J Med 2008, 359(4):366-377.

21. Watanabe M, Kawaguchi T, Isa S, Ando M, Tamiya A, Kubo A, Saka H, Takeo S, Adachi H, Tagawa T et al: Ultra-Sensitive Detection of the Pretreatment EGFR T790M Mutation in Non-Small Cell Lung Cancer Patients with an EGFR-Activating Mutation Using Droplet Digital PCR. Clin Cancer Res 2015, 21(15):3552-3560.

22. Oh JE, An CH, Yoo NJ, Lee SH: Detection of low-level EGFR T790M mutation in lung cancer tissues. APMIS 2011, 119(7):403-411.

23. Ye L, Mesbah Ardakani N, Thomas C, Spilsbury K, Leslie C, Amanuel B, Millward M: Detection of Lowlevel EGFR c.2369 C > T (p.Thr790Met) Resistance Mutation in Pre-treatment Non-small Cell Lung Carcinomas Harboring Activating EGFR Mutations and Correlation with Clinical Outcomes. Pathol Oncol Res 2020.

24. Lee SH, Kim EY, Kim A, Chang YS: Clinical implication and usefulness of de novo EGFR T790M mutation in lung adenocarcinoma with EGFR-tyrosine kinase inhibitor sensitizing mutation. Cancer Biol Ther 2020, 21(8):741-748.

25. Kobayashi S, Boggon TJ, Dayaram T, Janne PA, Kocher O, Meyerson M, Johnson BE, Eck MJ, Tenen DG, Halmos B: EGFR mutation and resistance of non-small-cell lung cancer to gefitinib. N Engl J Med 2005, 352(8):786-792.

26. Yun $\mathrm{CH}$, Mengwasser KE, Toms AV, Woo MS, Greulich H, Wong KK, Meyerson M, Eck MJ: The T790M mutation in EGFR kinase causes drug resistance by increasing the affinity for ATP. Proc Natl Acad Sci U S A 2008, 105(6):2070-2075.

27. Fujii A, Harada T, Iwama E, Ota K, Furuyama K, ljichi K, Okamoto T, Okamoto I, Takayama K, Nakanishi Y: Hypermethylation of the CpG dinucleotide in epidermal growth factor receptor codon 790: implications for a mutational hotspot leading to the T790M mutation in non-small-cell lung cancer. Cancer Genet 2015, 208(5):271-278. 
28. Mok TS, Wu YL, Ahn MJ, Garassino MC, Kim HR, Ramalingam SS, Shepherd FA, He Y, Akamatsu H, Theelen WS et al: Osimertinib or Platinum-Pemetrexed in EGFR T790M-Positive Lung Cancer. N Engl J Med 2017, 376(7):629-640.

29. Pinheiro LB, Coleman VA, Hindson CM, Herrmann J, Hindson BJ, Bhat S, Emslie KR: Evaluation of a droplet digital polymerase chain reaction format for DNA copy number quantification. Anal Chem 2012, 84(2):1003-1011.

30. Wang S, Yan B, Zhang Y, Xu J, Qiao R, Dong Y, Zhang B, Zhao Y, Zhang L, Qian J et al: Different characteristics and survival in non-small cell lung cancer patients with primary and acquired EGFR T790M mutation. International Journal of Cancer 2019, 144(11):2880-2886.

31. Tian P, Wang Y, Wang W, Li Y, Wang K, Cheng X, Tang Y, Han-Zhang H, Ye J, Chuai S et al: Highthroughput sequencing reveals distinct genetic features and clinical implications of NSCLC with de novo and acquired EGFR T790M mutation. Lung Cancer 2018, 124:205-210.

32. Li W, Qiu T, Guo L, Ling Y, Gao Y, Ying J, He J: Primary and acquired EGFR T790M-mutant NSCLC patients identified by routine mutation testing show different characteristics but may both respond to osimertinib treatment. Cancer Lett 2018, 423:9-15.

33. Chen L-Y, Molina-Vila MA, Ruan S-Y, Su K-Y, Liao W-Y, Yu K-L, Ho C-C, Shih J-Y, Yu C-J, Yang JC-H et al: Coexistence of EGFR T790M mutation and common activating mutations in pretreatment non-small cell lung cancer: A systematic review and meta-analysis. Lung Cancer 2016, 94:46-53.

34. Liang H, Pan Z, Wang W, Guo C, Chen D, Zhang J, Zhang Y, Tang S, He J, Liang W: The alteration of T790M between 19 del and L858R in NSCLC in the course of EGFR-TKIs therapy: a literature-based pooled analysis. Journal of Thoracic Disease 2018, 10(4):2311-2320.

35. Dong Y, Zhou Z, Wang J, Ma L, Liu Z, Wang Y, Song J, Zhang S, Che N: Origin of the T790M mutation and its impact on the clinical outcomes of patients with lung adenocarcinoma receiving EGFR-TKIs. Pathol Res Pract 2019, 215(5):946-951.

36. Ding D, Yu Y, Li Z, Niu X, Lu S: The predictive role of pretreatment epidermal growth factor receptor T790M mutation on the progression-free survival of tyrosine-kinase inhibitor-treated non-small cell lung cancer patients: a meta-analysis. Onco Targets Ther 2014, 7:387-393.

37. Hidaka N, Iwama E, Kubo N, Harada T, Miyawaki K, Tanaka K, Okamoto I, Baba E, Akashi K, Sasaki H et al: Most T790M mutations are present on the same EGFR allele as activating mutations in patients with non-small cell lung cancer. Lung Cancer 2017, 108:75-82.

38. Oxnard GR, Arcila ME, Chmielecki J, Ladanyi M, Miller VA, Pao W: New strategies in overcoming acquired resistance to epidermal growth factor receptor tyrosine kinase inhibitors in lung cancer. Clin Cancer Res 2011, 17(17):5530-5537.

39. Kuiper JL, Heideman DA, Thunnissen E, Paul MA, van Wijk AW, Postmus PE, Smit EF: Incidence of T790M mutation in (sequential) rebiopsies in EGFR-mutated NSCLC-patients. Lung Cancer 2014, 85(1):19-24.

40. Matsuo N, Azuma K, Sakai K, Hattori S, Kawahara A, Ishii H, Tokito T, Kinoshita T, Yamada K, Nishio K et al: Association of EGFR Exon 19 Deletion and EGFR-TKI Treatment Duration with Frequency of 
T790M Mutation in EGFR-Mutant Lung Cancer Patients. Sci Rep 2016, 6:36458.

41. Ramirez M, Rajaram S, Steininger RJ, Osipchuk D, Roth MA, Morinishi LS, Evans L, Ji W, Hsu C-H, Thurley $\mathrm{K}$ et al: Diverse drug-resistance mechanisms can emerge from drug-tolerant cancer persister cells. Nature Communications 2016, 7(1).

42. Lantermann AB, Chen D, McCutcheon K, Hoffman G, Frias E, Ruddy D, Rakiec D, Korn J, McAllister G, Stegmeier $\mathrm{F}$ et al: Inhibition of Casein Kinase 1 Alpha Prevents Acquired Drug Resistance to Erlotinib in EGFR-Mutant Non-Small Cell Lung Cancer. Cancer Res 2015, 75(22):4937-4948.

43. Cragg MS, Kuroda J, Puthalakath $\mathrm{H}$, Huang DC, Strasser A: Gefitinib-induced killing of NSCLC cell lines expressing mutant EGFR requires BIM and can be enhanced by BH3 mimetics. PLoS Med 2007, 4(10):1681-1689; discussion 1690.

44. Tanimoto A, Takeuchi S, Arai S, Fukuda K, Yamada T, Roca X, Ong ST, Yano S: Histone Deacetylase 3 Inhibition Overcomes BIM Deletion Polymorphism-Mediated Osimertinib Resistance in EGFR-Mutant Lung Cancer. Clin Cancer Res 2017, 23(12):3139-3149.

45. Zheng D, Ye X, Zhang MZ, Sun Y, Wang JY, Ni J, Zhang HP, Zhang L, Luo J, Zhang J et al: Plasma EGFR T790M ctDNA status is associated with clinical outcome in advanced NSCLC patients with acquired EGFR-TKI resistance. Sci Rep 2016, 6:20913.

46. Remon J, Menis J, Hasan B, Peric A, De Maio E, Novello S, Reck M, Berghmans T, Wasag B, Besse B et al: The APPLE Trial: Feasibility and Activity of AZD9291 (Osimertinib) Treatment on Positive PLasma T790M in EGFR-mutant NSCLC Patients. EORTC 1613. Clin Lung Cancer 2017, 18(5):583588.

\section{Tables}

Table 1. Clinicopathological characteristics of the patients with EGFR mutation 


\begin{tabular}{|c|c|c|c|c|c|c|}
\hline \multirow[t]{2}{*}{ Characteristics } & \multirow{2}{*}{$\begin{array}{l}\text { Total subjects } \\
(n=59)\end{array}$} & \multicolumn{2}{|c|}{ EGFR+/T790M+ $(n=17)$} & \multicolumn{2}{|c|}{ EGFR+/T790M- $(n=42)$} & \multirow[t]{2}{*}{ P-value } \\
\hline & & No. of patients & $\%$ & No. of patients & $\%$ & \\
\hline \multicolumn{7}{|l|}{ Gender } \\
\hline Male & 28 & 8 & 28.6 & 20 & 71.4 & 1.000 \\
\hline Female & 31 & 9 & 29.0 & 22 & 71.0 & \\
\hline \multicolumn{7}{|l|}{ Age (years) } \\
\hline$>60$ & 25 & 9 & 36.0 & 16 & 64.0 & 0.386 \\
\hline$\leq 60$ & 34 & 8 & 23.5 & 26 & 76.5 & \\
\hline \multicolumn{7}{|l|}{ ECOG-PS score } \\
\hline $0-1$ & 56 & 15 & 26.8 & 41 & 73.2 & 0.197 \\
\hline$\geq 2$ & 3 & 2 & 66.7 & 1 & 33.3 & \\
\hline \multicolumn{7}{|l|}{ Smoking status } \\
\hline Yes & 10 & 2 & 20.0 & 8 & 80.0 & 0.708 \\
\hline No & 49 & 15 & 30.6 & 34 & 69.4 & \\
\hline \multicolumn{7}{|l|}{ Brain metastases } \\
\hline Yes & 19 & 6 & 31.6 & 13 & 68.4 & 0.766 \\
\hline No & 40 & 11 & 27.5 & 29 & 72.5 & \\
\hline \multicolumn{7}{|l|}{ Stage } \\
\hline $\mathbb{\nabla A}$ & 19 & 8 & 42.1 & 11 & 57.9 & 0.781 \\
\hline$\triangle \mathrm{B}$ & 40 & 9 & 22.5 & 31 & 77.5 & \\
\hline \multicolumn{7}{|l|}{ Histology } \\
\hline Adenocarcinoma & 58 & 17 & 29.3 & 41 & 70.7 & 1.000 \\
\hline Adenosquamous & 1 & 0 & 0 & 1 & 100 & \\
\hline \multicolumn{7}{|l|}{ EGFR mutations } \\
\hline 19del & 34 & 14 & 41.2 & 20 & 58.8 & 0.020 \\
\hline 21L858R & 25 & 3 & 12.0 & 22 & 88.0 & \\
\hline
\end{tabular}

\section{Figures}




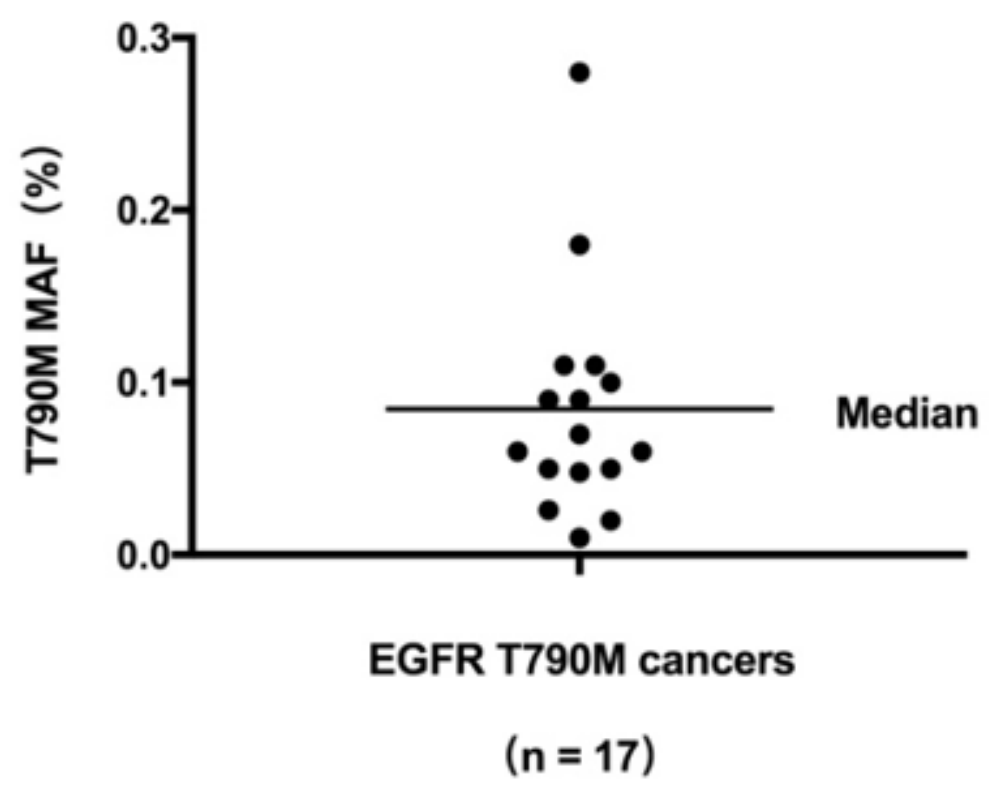

Figure 1

Detection of EGFR T790M mutation alleles in ctDNA samples from pretreatment NSCLC patients with EGFR-activating mutation. Analysis of the frequency of alleles in 17 NSCLC patients with de novo T790M mutation showed the MAF ranged from $0.01 \%$ to $0.28 \%$.

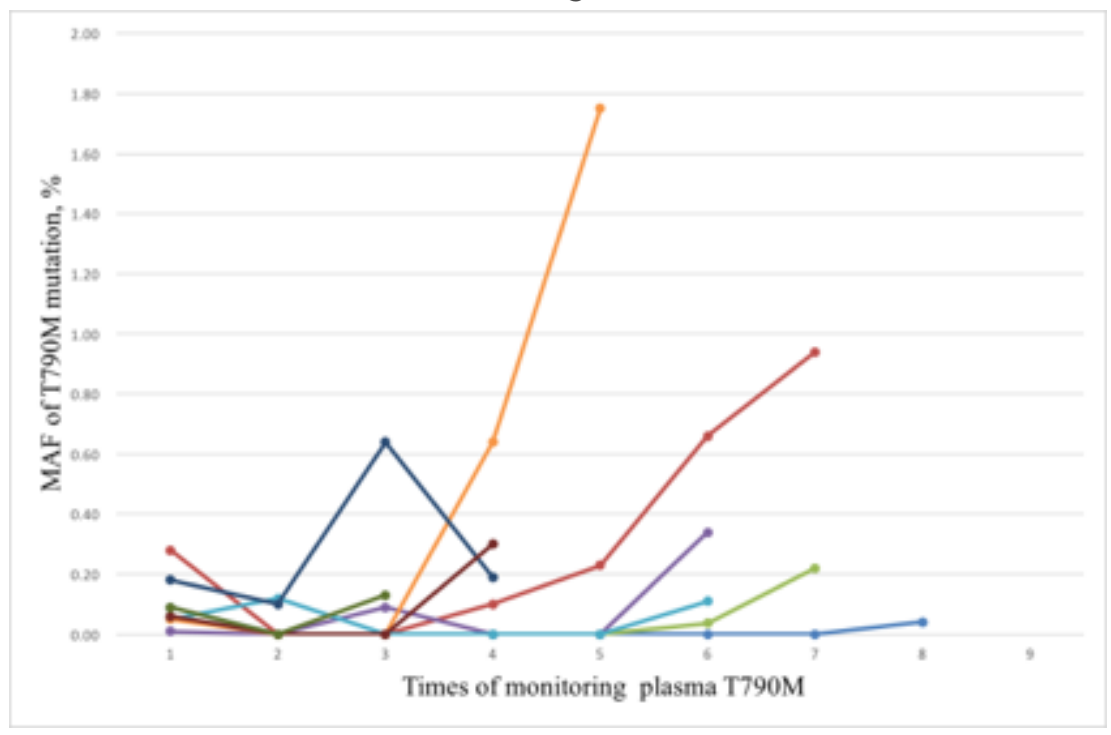

Figure 2

Monitoring plasma T790M mutation in patients with low-frequency de novo T790M mutation during treatment with first-generation EGFR-TKIs. An upward trend in the MAF of T790M mutation was observed in nine patients after tumor progression. 


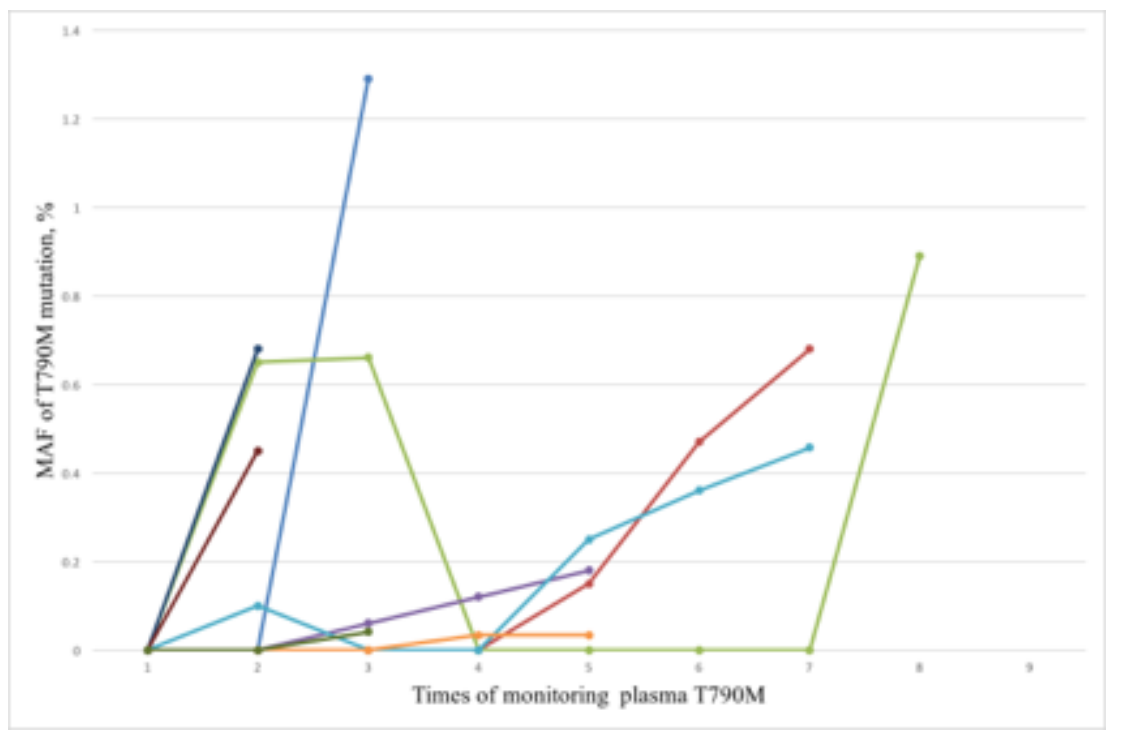

\section{Figure 3}

Monitoring plasma T790M mutation during first-generation EGFR-TKI treatment in pre-treatment T790Mnegative patients. The MAF of T790M mutation in nine patients over the course of tumor development showed an upward trend.

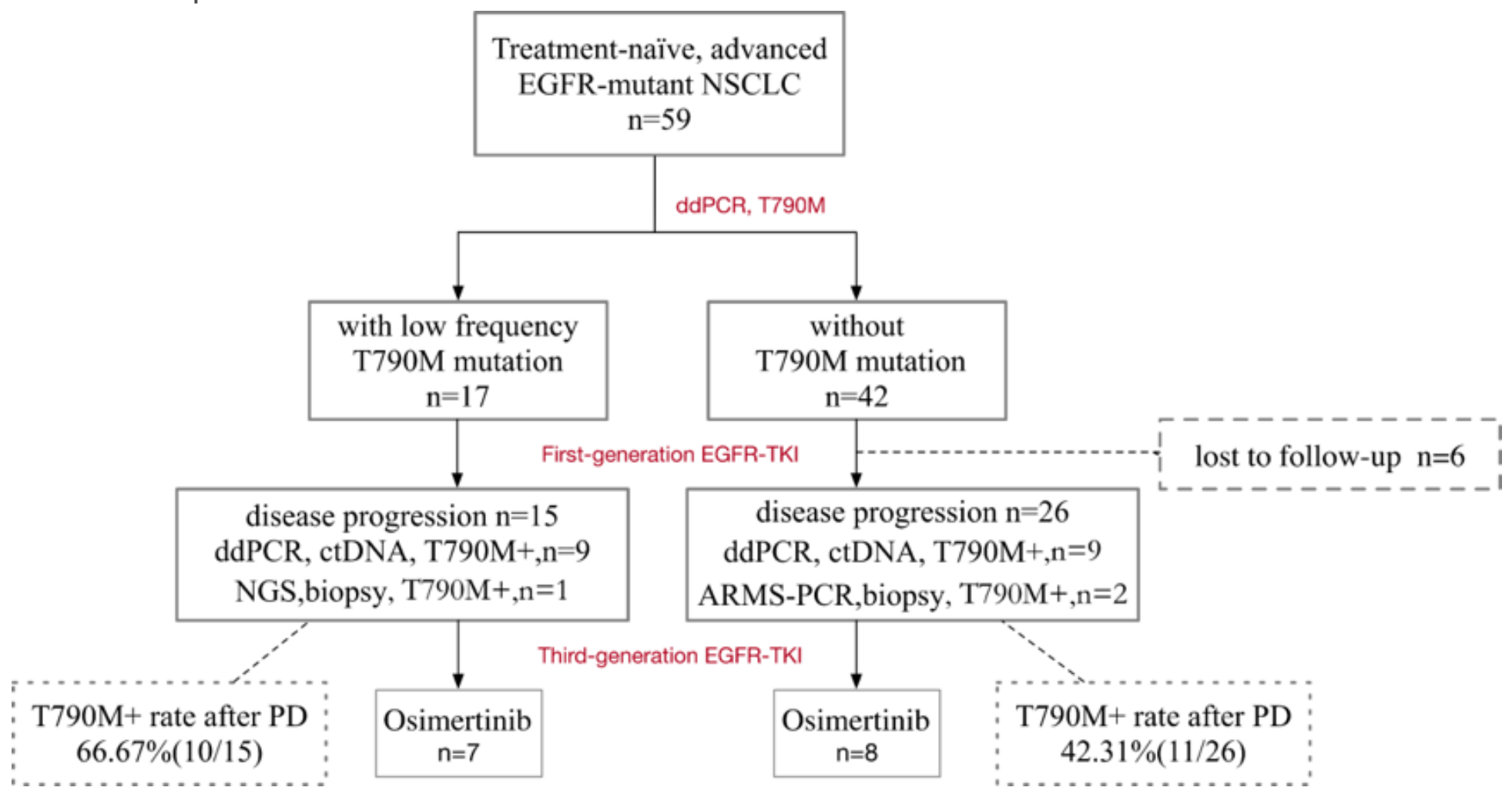

\section{Figure 4}

Process flowchart for monitoring the EGFR T790M mutation in patients during EGFR-TKI treatment. 


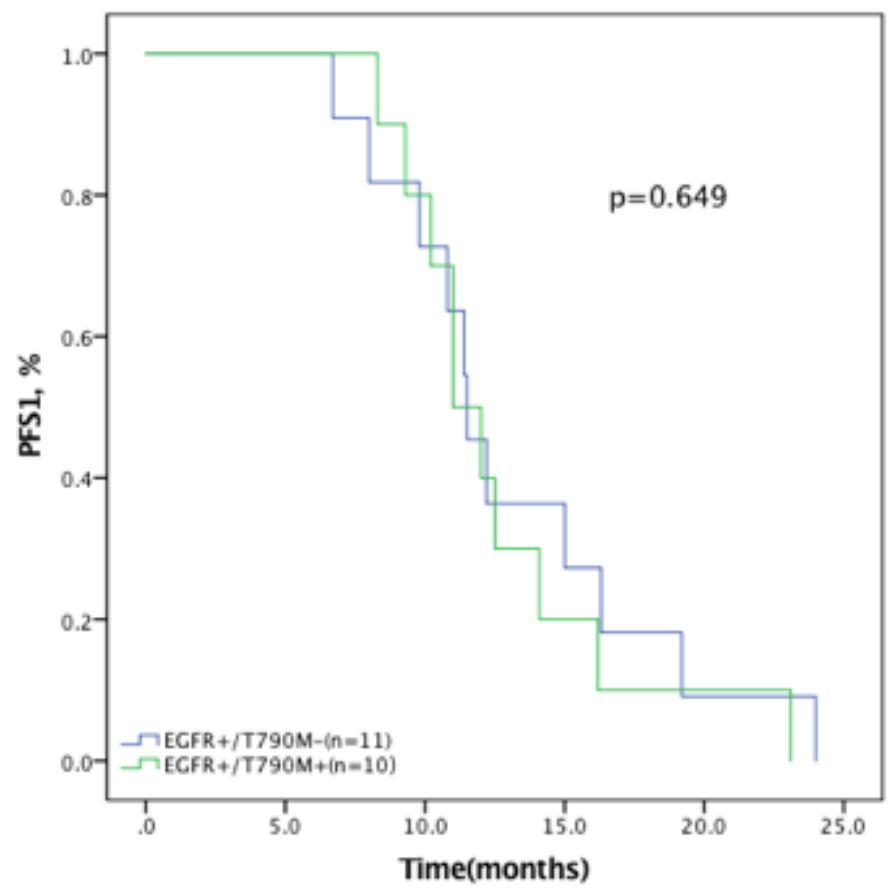

\section{Figure 5}

Kaplan-Meier curves for PFS1 in patients with T790M resistance mutation after disease progression during first-generation EGFR-TKI treatment. Comparison of the median PFS1 between the patients with low-frequency de novo T790M mutation and those without pretreatment T790M mutation did not show a significant difference between the two groups (11.0 months VS. 11.5 months; $p=0.649$ ).

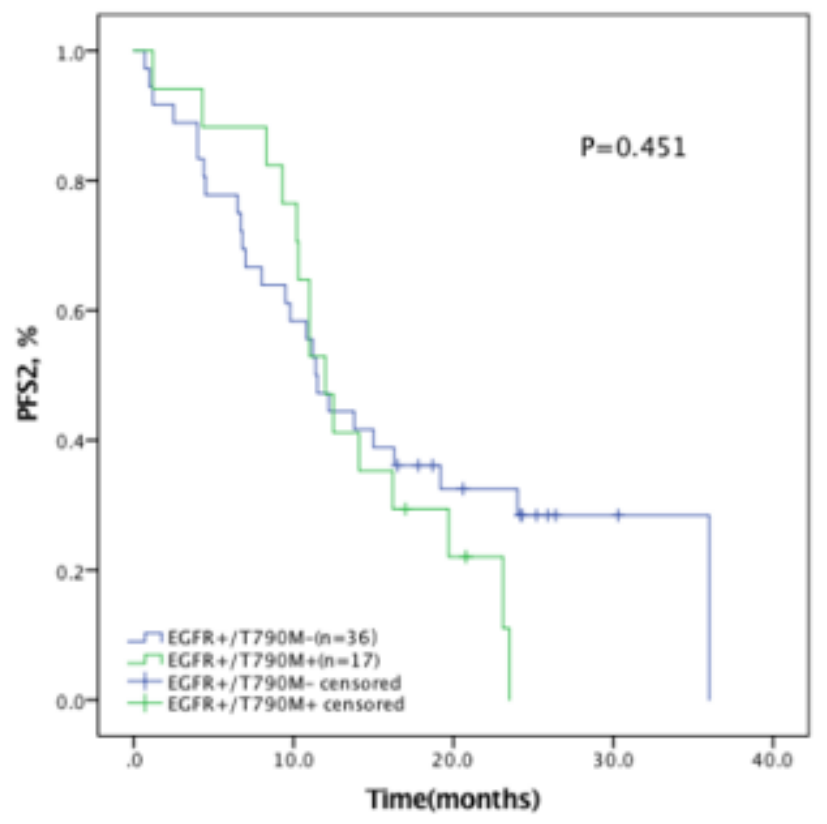

Figure 6 
Kaplan-Meier curves for PFS2 for advanced EGFR-activating NSCLC patients with and without de novo T790M mutation during first-generation EGFR-TKI treatment. The median PFS2 was comparable between the patients with and without low-frequency de novo T790M mutation before treatment (12.0 months VS. 11.4 months, respectively; $p=0.451$ ).

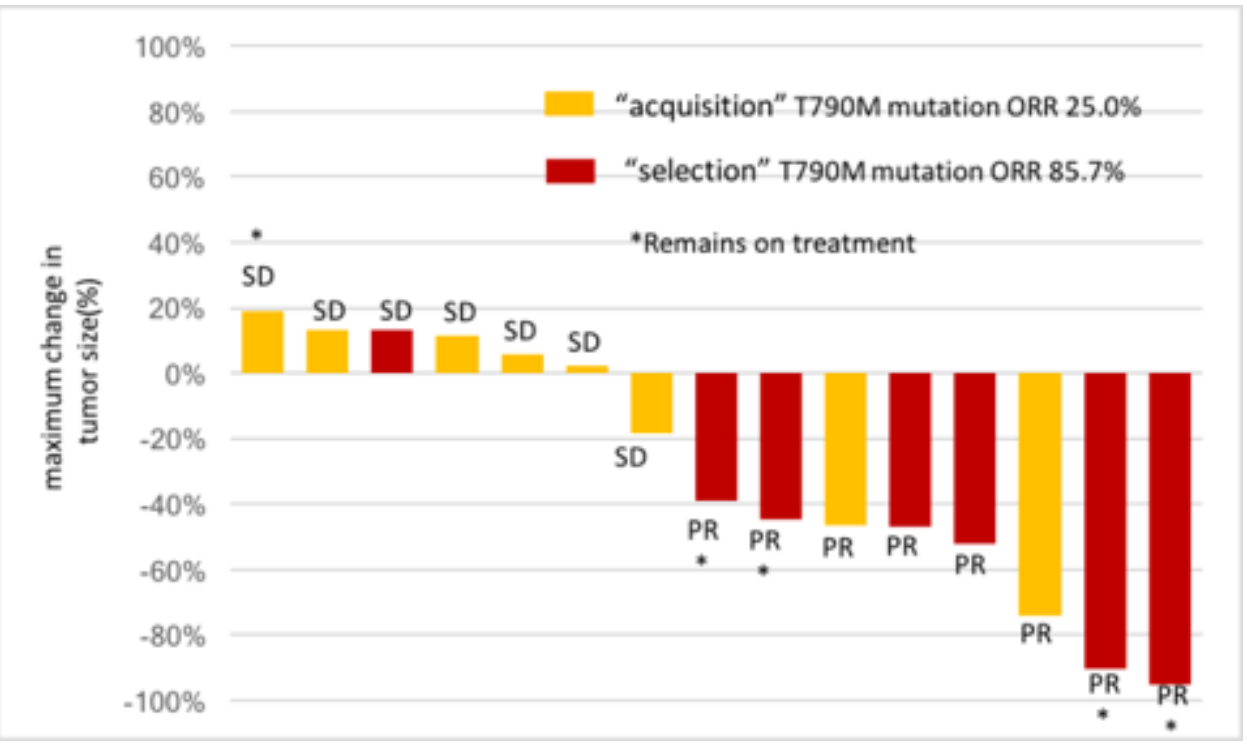

\section{Figure 7}

The efficacy of the third-generation EGFR-TKI for patients with "selection" and "acquisition" T790M resistance mutations. The red histogram represents the patients with the "selection" T790M resistance mutation, among which the ORR was $85.7 \%$. The yellow histogram represents the patients with the "acquisition" T790M resistance mutation and their ORR was 25.0\%. (partial response=PR, stable disease $=S D$, objective response rate $=\mathrm{ORR}$ )

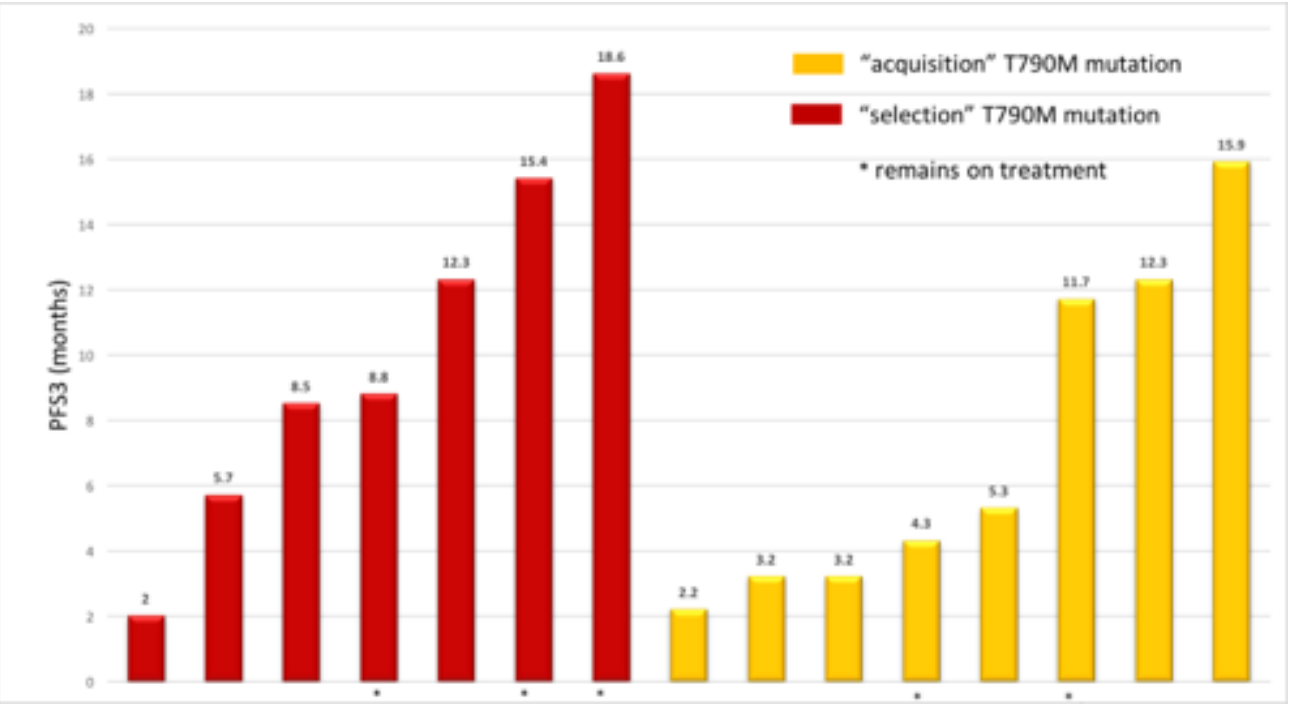

Figure 8

PFS3 after third-generation EGFR-TKI treatment for patients with "selection" and "acquisition" T790M resistance mutations. The red histogram represents the patients with the "selection" T790M resistance 
mutation; the PFS3 for five patients was more than 8 months in this group. The yellow histogram represents the patients with the "acquisition" T790M resistance mutation; the PFS3 for five patients was less than 6 months in this group. 